\title{
An unusual case of gestational diabetes mellitus
}

\author{
P Shankar, M K Sundarka, A Sundarka
}

Postgrad Med J 2002;78:562-563

The case of a woman, who developed gestational diabetes at six weeks in two successive pregnancies, is reported. She was in the high risk group to develop gestational diabetes. The first pregnancy terminated in abortion at 10 weeks. In the second pregnancy, institution of insulin therapy at diagnosis was associated with a successful outcome. This case highlights the need for screening for gestational diabetes at the first antenatal visit in the high risk group.

A 29 year old Asian woman, a nurse by profession, presented to our diabetes clinic at six weeks in her first pregnancy in June 2000. Her body mass index was 23 and blood pressure was 130/80 $\mathrm{mm} \mathrm{Hg}$. There was no relevant past medical history. There was a strong family history of type 2 diabetes as both her parents were diabetic and one sister had gestational diabetes and later developed type 2 diabetes. Our patient was worried about her glycaemic status and to allay her fears, a $75 \mathrm{~g}$ oral glucose tolerance test (OGTT) was performed. The fasting plasma glucose was $6.3 \mathrm{mmol} / \mathrm{l}$ and two hour value was $8.1 \mathrm{mmol} / \mathrm{l}$. On the basis of the World Health Organisation 1999 diagnostic criteria, gestational diabetes mellitus was diagnosed and necessary dietary advice given. ${ }^{1}$ Unfortunately, the pregnancy terminated in abortion at 10 weeks. Eight weeks after the abortion a $75 \mathrm{~g}$ OGTT was repeated, which was normal. She was advised to follow a normal diet and to undergo an OGTT as soon as the next pregnancy was detected.

She conceived again in February 2001 and underwent a $75 \mathrm{~g}$ OGTT at six weeks' gestation. The fasting value was $5.7 \mathrm{mmol} / \mathrm{l}$ and two hour value was $10 \mathrm{mmol} / \mathrm{l}$. Glycated haemoglobin $\left(\mathrm{HbA}_{\mathrm{Ic}}\right)$ done at the same time was 6.6\% (reference range $6.6 \%-8.3 \%)$. The diagnosis of gestational diabetes mellitus was made and insulin therapy in the form of twice daily injections of human biphasic isophane insulin (Huminsulin 30/70, Eli Lilly) was started. Follow ups were done at intervals of 2-4 weeks depending on the glycaemic control achieved. Her average fasting and two hour plasma glucose values were 4.3 $\mathrm{mmol} / \mathrm{l}$ and $6.8 \mathrm{mmol} / \mathrm{l}$ respectively with average $\mathrm{HbA}_{\mathrm{1c}}$ being less than $6.5 \%$. The insulin doses needed increasing with advancing pregnancy. At the end of the pregnancy, the daily insulin dose was 30 units. The weight gain during pregnancy was $13 \mathrm{~kg}$. At 37 weeks, the pregnancy was complicated by premature rupture of the membranes. Induction of labour was complicated by fetal bradycardia necessitating an emergency caesarean section. A live and healthy female baby was delivered weighing $2850 \mathrm{~g}$. The baby had an uncomplicated neonatal course. The patient's plasma glucose values returned to normal by the next morning and insulin injections were stopped. She underwent a $75 \mathrm{~g}$ OGTT at six weeks postpartum, which was normal. She was informed of her high risk of developing type 2 diabetes in later life, ${ }^{2}$ and was advised to maintain normal body weight by appropriate dietary modifications and regular physical activity to prevent this. She was also advised to undergo a 75 g OGTT every three years. ${ }^{2}$

\section{DISCUSSION}

Gestational diabetes mellitus is a heterogeneous entity that includes women with pregnancy induced glucose intolerance and women with undiagnosed diabetes discovered during pregnancy. ${ }^{2}$ Normal pregnancy is characterised by mild fasting hypoglycaemia, a moderate rise in postprandial plasma glucose, and hyperinsulinaemia. ${ }^{3}$ The postprandial hyperglycaemia is due to pregnancy induced physiological insulin resistance. ${ }^{4}$ Although the underlying mechanism(s) for this are not completely understood, hormones like cortisol, prolactin, progesterone, and human placental lactogen may be involved. ${ }^{4}$ The most likely candidate is human placental lactogen because of 1000-fold rise in its level during pregnancy and its homology to the known insulin antagonist growth hormone. ${ }^{4}$ As the level of these hormones rise with advancing pregnancy, so the insulin resistance worsens with time. ${ }^{4}$ It is at its maximum in the third trimester, necessitating a threefold rise in maternal insulin output to maintain euglycaemia. ${ }^{4}$ Mothers with deficient $\beta$ cell reserve become glucose intolerant at this time. ${ }^{4}$ So, gestational diabetes mellitus typically appears late in the second trimester or early in the third trimester. ${ }^{4}$ Based on this fact, the current recommendation for screening for gestational diabetes mellitus is between 24 and 28 weeks' gestation. ${ }^{13}$

Our patient became glucose intolerant at six weeks in two successive pregnancies and was normoglycaemic outside these pregnancies. It can be concluded that the underlying cause of her glucose intolerance was early onset gestational diabetes rather than pregestational diabetes. Decreased peripheral insulin sensitivity is the most probable explanation for her early onset gestational diabetes as evident by a landmark prospective study done by Catalano et al..$^{5}$ They evaluated longitudinal changes in peripheral insulin sensitivity in women before and during a planned pregnancy with the help of the hyperinsulinaemic-euglycaemic clamp and infusion of $\left[6,6^{2} \mathrm{H}_{2}\right]$ glucose and were able to demonstrate that the women who developed gestational diabetes mellitus had decreased peripheral insulin sensitivity even before conception and in early pregnancy (at 12-14 weeks' gestation). ${ }^{5}$

Although early onset gestational diabetes mellitus-that is, glucose intolerance developing before 24 weeks' gestation-is a newly described entity, it accounts for a significant proportion of total cases as demonstrated in a study by Bartha et al. ${ }^{6}$ In their follow up of 3986 pregnant women, 30\% (65) cases of gestational diabetes were diagnosed early in pregnancy. ${ }^{6}$ Out of these $65,61.5 \%$ (40) were diagnosed between 6 and 13 weeks of gestation. ${ }^{6}$ The subgroup of women with early onset gestational diabetes was at a higher risk to develop hypertension and pre-eclampsia compared with the subgroup of women with late onset gestational diabetes. ${ }^{6}$ There was a higher incidence of neonatal hypoglycaemia and perinatal death and these women were more likely to require

Abbreviations: $\mathrm{HbA}_{\mathrm{lc}}$, glycated haemoglobin; OGTT, oral glucose tolerance test 
insulin for glycaemic control. ${ }^{6}$ It was likely that early intervention was beneficial, as no fetal anomalies were observed, and the rates of hydromnios and preterm labour were similar between the two groups. ${ }^{6}$ In another study, the same investigators demonstrated that women with early onset gestational diabetes had higher risk of developing postpartum diabetes and glucose intolerance.

Although our patient belonged to high risk group to develop gestational diabetes, we would not have screened her before 24 weeks' gestation if she were not insistent. This case calls for modifying the present clinical practice when dealing with pregnant women at high risk to develop gestational diabetes. The American Diabetes Association in its position statement (2001) advocates screening of the high risk group for gestational diabetes at the first antenatal visit. ${ }^{2}$ If they are found not to have gestational diabetes mellitus at the initial screening, they should be retested between 24 and 28 weeks of gestation. ${ }^{2}$ The World Health Organisation guidelines (1999) have the same recommendation. ${ }^{1}$

\section{Authors' affiliations}

P Shankar, M K Sundarka, Department of Medicine, Manipal College of Medical Sciences-Teaching Hospital, Pokhara, Nepal

A Sundarka, Department of Obstetrics and Gynaecology

Correspondence to: Dr Praveen Shankar, Department of Medicine, Manipal Teaching Hospital, Pokhara, Nepal; prvshankarus@yahoo.com

Submitted 14 January 2002

Accepted 19 June 2002

\section{Learning points}

- Early onset gestational diabetes mellitus constitutes a high risk subgroup in women with gestational diabetes.

- In women at high risk of developing gestational diabetes mellitus, screening at the first antenatal visit is advisable.

- Early onset gestational diabetes mellitus should be managed in the same way as pregestational diabetes.

- Women with early onset gestational diabetes mellitus are at a higher risk of developing postpartum glucose intolerance and diabetes mellitus.

\section{REFERENCES}

1 World Health Organisation. Definition, diagnosis and classification of diabetes mellitus and its complications. Part 1. Diagnosis and classification of diabetes mellitus. Geneva: World Health Organisation, 1999.

2 American Diabetes Association. Gestational diabetes mellitus. Diabetes Care 2001;24(suppl 1):S77-9.

3 Inzucchi SE. Diabetes in pregnancy. In: Burrow GN, Duffy TP, eds. Medical complications during pregnancy. 5th Ed. Philadelphia: WB Saunders, 1999: 25-51.

4 Ryan EA. Pregnancy in diabetes. Med Clin North Am 1998;82:823-45

5 Catalano PM, Tyzbir ED, Wolfe RR, et al. Carbohydrate metabolism during pregnancy in control subjects and women with gestational diabetes. Am J Physiol 1993;264:E60-70.

6 Bartha JL, Martinez-Del-Fresno P, Comino-Delgado R. Gestational diabetes diagnosed during early pregnancy. Am J Obstet Gynecol $2000 ; 182: 346-50$

7 Bartha JL, Martinez-Del-Fresno P, Comino-Delgado R. Postpartum metabolism and autoantibody markers in women with gestational diabetes diagnosed in early pregnancy. Am J Obstet Gynecol $2001 ; 184: 965-70$.

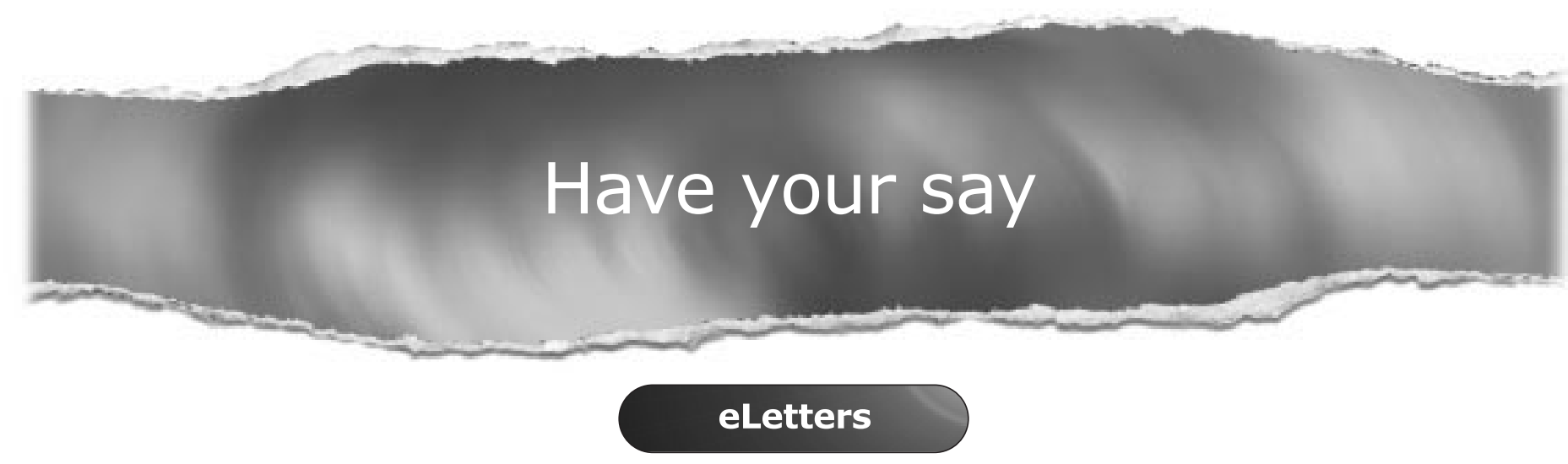

If you wish to comment on any article published in the Postgraduate Medical Journal you can send an eLetter using the eLetters link at the beginning of each article. Your response will be posted on Postgraduate Medical Journal online within a few days of receipt (subject to editorial screening).

\section{www.postgradmedj.com}

